\title{
Design of Pressure Sensor Based on Two-Dimensional Photonic Crystal
}

\author{
T. Zouache*, A. Hocini, A. Harhouz, R. Mokhtari \\ Laboratoire d'Analyse des Signaux et Systèmes, Electronics Department P. 166, Route Ichebilia, \\ Université Mohamed Boudiaf de M'sila, M'sila 28000, Algeria
}

\begin{abstract}
In this work, we design a new pressure sensor based on two-dimensional photonic crystal waveguide coupled to a point-defect resonant microcavity. The mechanism of sensing is based on the change of the germanium refractive index as function of the hydrostatic pressure $P$. The resonant wavelength will shift when pressure variation induces change in the refractive indexes of the structure. The pressure variation causes the shifting of defect modes. The properties of the refractive index sensor are simulated using the finite-difference time-domain algorithm and the plane wave expansion method. These kinds of sensors have many advantages in compactness, high sensitivity, and various choices of materials.
\end{abstract}

DOI: 10.12693/APhysPolA.131.68

PACS/topics: 42.70.Qs, 78.67.Pt, 07.07.Df, 42.79.--e

\section{Introduction}

The photonic crystal $(\mathrm{PhC})$ explores a new research direction in optical sensing field. Photonic crystal is a periodic dielectric structure with lattice parameter on the order of wavelength of propagated electromagnetic wave. One of very important characteristics of photonic crystal is its light confinement and controlling property. These characteristics allowed the crystal to use in various sensing applications [1-3]. The photonic crystal sensors has received considerable attention in developing label-free pressure sensor, besides, refractive index (RI) change is directly linked to the existence of the force onto a sensing surface of the device, the refractive index sensor can realize sensitive detection with small sample volumes.

In this work, we design new pressure sensor based on a 2D photonic crystal waveguide incorporating with microcavity to sense small refractive index changes. The proposed RI sensor is formed with waveguides and a microcavity in the $\mathrm{PhC}$ with a triangular lattice of air holes. The sensing principle is based on the shift of resonance wavelength, which occurs due to the change in RI of the sensor when the germanium PhC's refractive index is changed by the application of a hydrostatic pressure onto a sensing surface. The sensor is appropriate for detecting homogeneous pressure. A theoretical model is developed to evaluate the change of the refractive index of $2 \mathrm{D}$ photonic crystal induced by the application of the pressure onto a sensing surface. The properties of the sensor are simulated using the finite-difference time-domain (FDTD) algorithm and the plane wave expansion (PWE) method. Based on a previous paper [4], the present paper describes a new kind of pressure sensor using germanium slab, since this material is compatible with the standard CMOS processing of the microelectronics industry.

*corresponding author; e-mail: T.Zouache@univ-msila.dz

\section{2D sensor design}

The structure presented in this work consists of a periodic disposition of $31 \times 31$ matrix of air holes $\left(n_{\text {air }}=1\right)$ with a radius of $r=0.43 a$ in a germanium $(\mathrm{Ge})$ slab, this structure is organized in a triangular lattice with a spatial period, $a=550 \mathrm{~nm}$ (with index profile of germanium slab $\left(n_{\mathrm{Ge}} \approx 4.0\right)$ and air $\left.\left(n_{\mathrm{air}}=1\right)\right)$ (Fig. 1a). Utilizing the triangular PhCs is practically important since they have a large band gap (the largest band gap for transverse electric (TE) polarization in the connected structure, and transverse magnetic (TM) in the deconnected structure) and it is expected to serve as a good platform for photonic integrated circuits and ultra-compact optical sensors [5]. Figure 1b shows a preliminary analysis of dispersion diagram showing normalized frequency versus the wave vector for TM modes of the $2 \mathrm{D}$ photonic crystal. It has been calculated along the $\Gamma-K-M-\Gamma$ edge for the Brillouin zone by employing a 2D PWE method of the RSoft (Band SOLVE) software. The regular PhC structure exhibits a large band gap between $1271.2 \mathrm{~nm}$ and $2286.6 \mathrm{~nm}$ for TM polarization.

In photonic crystal with proper periodicity no wavelength is allowed to propagate in the range of photonic band gap (PBG). The localization of light can be obtained in the range of PBG by inducing defect (cavity and waveguide) [1].

Our proposed pressure sensor is formed with waveguide incorporating with microcavity. The waveguide is obtained by removing one row of air holes in the $\Gamma K$ direction. The microcavity is obtained by removing only one hole near the wave guide as shown in Fig. 1a. The incident source is a Gauss impulse light with a wave length of $1.55 \mu \mathrm{m}$ (which is a standard WL for telecom devices) located at the input of the waveguide and the monitor is placed at the end of the waveguide.

The sensor exploits the variation of the germanium refractive index, when a hydrostatic pressure $P$ is applied to its sensitive surface (Fig. 2). The relation between 

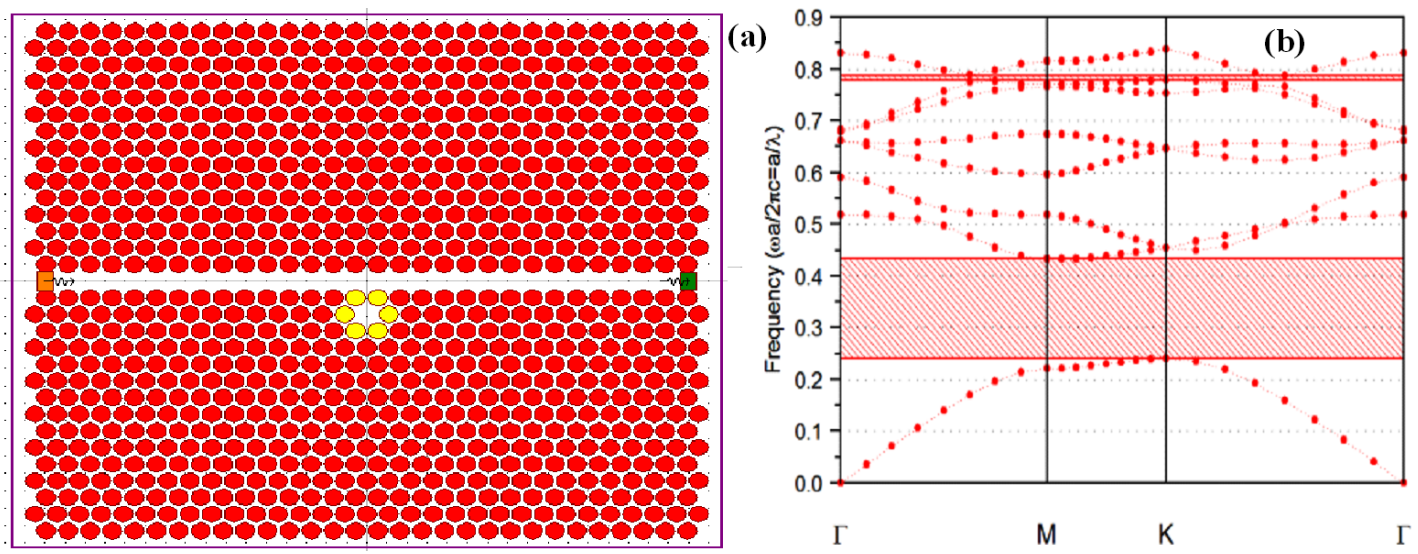

Fig. 1. (a) The designed pressure sensor, (b) calculated photonic band gap.

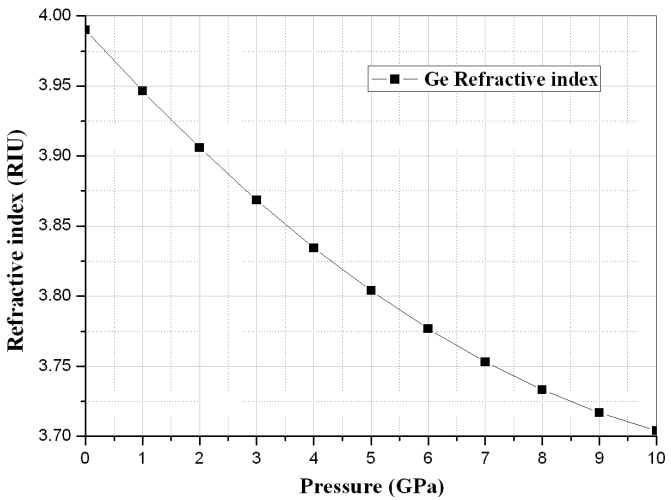

Fig. 2. Ge refractive index as a function of pressure.

hydrostatic pressure $P$ and germanium relative permittivity is given by [6]:

$$
n_{\mathrm{Ge}}=\sqrt{\varepsilon}_{r \mathrm{Ge}}=\sqrt{15.94-0.36 P+0.014 P^{2}} .
$$

This relation allows to have the $\varepsilon_{r \text { Ge }}$ of germanium known at ambient pressure given by literature $[7,8]$ with an accuracy of $5 \%$.

Using the relation (1) we draw the variation of the germanium refractive index for a range of pressure from $0 \mathrm{GPa}$ to $10 \mathrm{GPa}$. The results are shown in Fig. 2.

This curve shows a low non-linearity dependence of refractive index as a function of applied pressure. This means that we can use the considered range of pressure in our sensor simulation.

Figure 3 shows the transmittance obtained when exciting only the wave guide (red curve) and then when exciting the microcavity (black curve) for a RI of germanium corresponding to a $0 \mathrm{GPa}$ pressure. The response of the sensor for this last case is a peak located at $\lambda_{0}=1566.65 \mathrm{~nm}$ which corresponds to its response for an ambient pressure. This curve is very important because all shifts of $\lambda$ corresponding to the non-ambient applied pressures are measured with respect to $\lambda_{0}$ positions.

Now, knowing the $\lambda_{0}$ position we draw our sensor response for a pressure variation from $0 \mathrm{GPa}$ to $10 \mathrm{GPa}$ with 1 GPa step and the results are presented in Fig. 4a. This figure shows the existence of a $\lambda$ shifting when

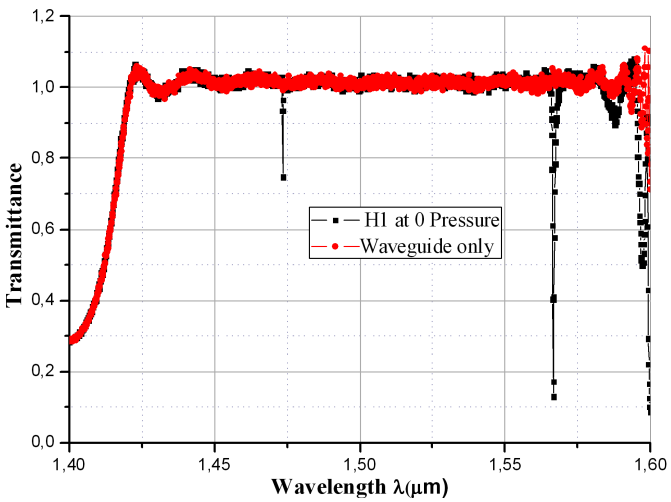

Fig. 3. Transmittance of the sensor at 0 pressure.

pressures change. This result is also shown in Fig. 4b. From Fig. 4a,b we can see clearly that the response of the designed sensor has a good transmittance and also for the region corresponding to high $P$, the peak positions are narrower. This can be explained by the existence of a relatively strong non-linearity in the $n_{\mathrm{Ge}}$ vs. pressure curve for this value of $P$.

The microcavity gives a shift of $15.8 \mathrm{~nm}$, equivalent to the sensitivity of $362.38 \mathrm{~nm} / \mathrm{RIU}$ for a change in RI value of 0.0436 (which corresponds to the change of $1 \mathrm{GPa}$ of pressure, Fig. 4a). For RI measurements, the sensitivity $(S)$ is calculated as the ratio of resonance wavelength $\lambda_{0}$ shift and the change in the RI and its unit is given by $\mathrm{nm} / \mathrm{RIU}$.

The sensitivity is the most important performance indicator that is worth investigating. It is calculated as the ratio of resonant wavelength shift and the change in the pressure and its unit is given by $\mathrm{nm} / \mathrm{GPa}$. It depends on the shift in resonant wavelength; the more shift is, the higher is the sensitivity. It is obtained from the relation

$$
\Delta S_{P}=\frac{\Delta \lambda}{\Delta P}=\frac{\Delta \lambda}{\Delta n} \frac{\Delta n}{\Delta P} .
$$

The obtained results are shown in Fig. 5. From this figure we see clearly that the sensitivity of sensor can reach $15.8 \mathrm{~nm} / \mathrm{GPa}$, the results show that the sensitivity decreases and reaches the lowest value 

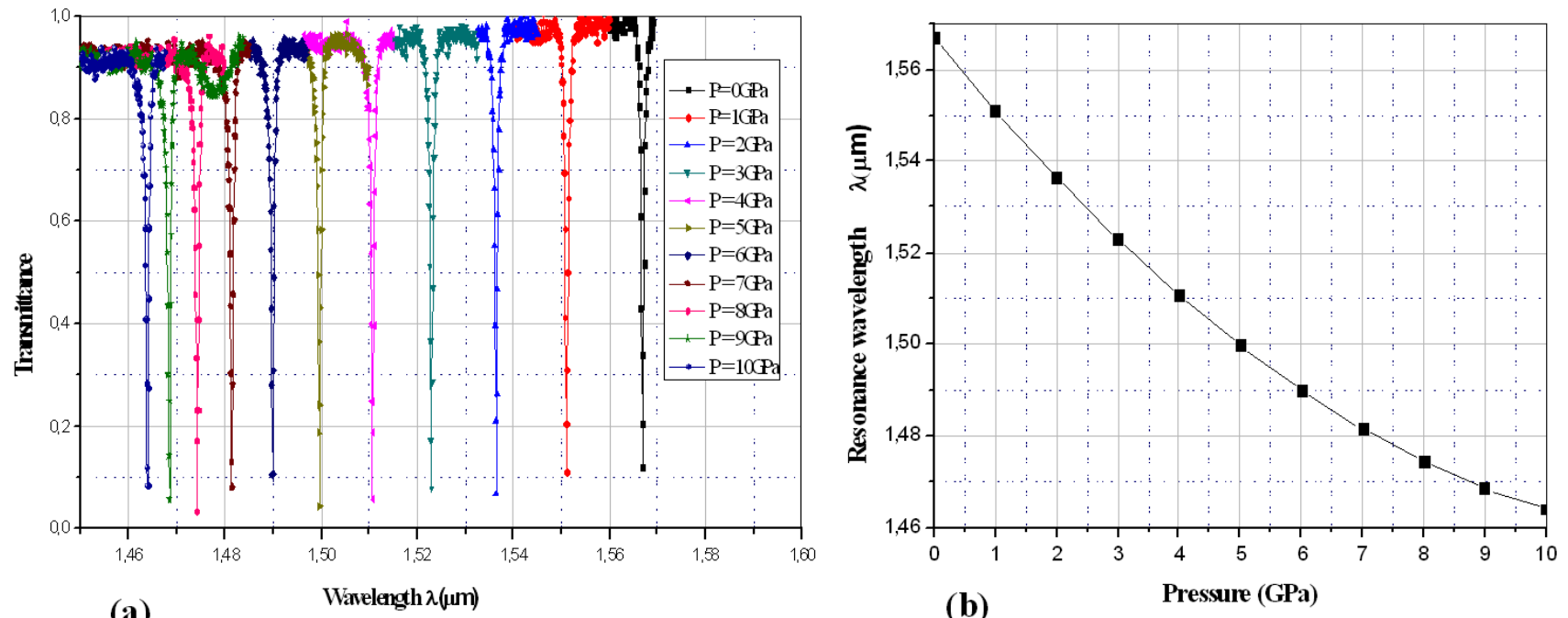

Fig. 4. (a) Transmittance for different applied pressure within the range 0-10 GPa. (b) The pressure vs. the output drop wavelength.

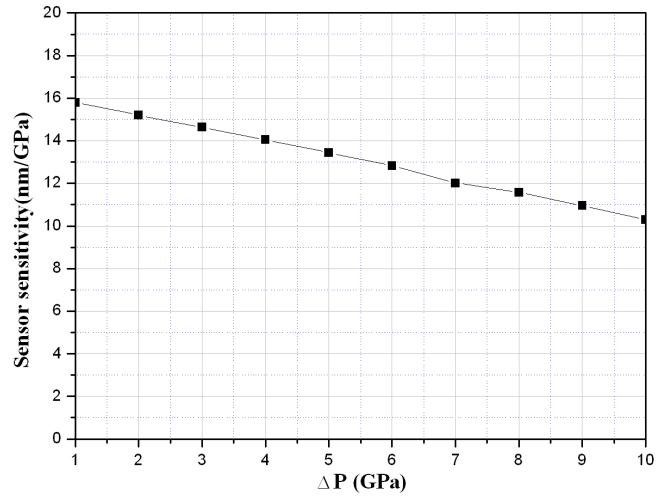

Fig. 5. Sensitivity $s$ variation with a change of pressure.

of sensitivity $10.15 \mathrm{~nm} / \mathrm{GPa}$. Comparing with other pressure sensor using $\mathrm{PhCs}$ which has a sensitivity of about $13.9 \mathrm{~nm} / \mathrm{GPa}$ under the dynamic range between 0 and $2 \mathrm{GPa}$ [9] our designed sensor has higher sensitivity.

\section{Conclusion}

In this paper we propose a new design of a $\mathrm{PhC}$ strainsensitive device (pressure sensor) consisting of a $\mathrm{PhC}$ waveguide $(\mathrm{PhCW})$ coupled to a $\mathrm{PhC}$ microcavity obtained by creating a point defect in the regular $2 \mathrm{D}$ periodic pattern based on germanium slab. The refractive index of Ge changes along with the applied pressure due to the photo-elastic effect. Through simulation, we have found that the resonant wavelength shifts and follows a linear law. The designed sensor detects pressure from $0 \mathrm{GPa}$ to $10 \mathrm{GPa}$. The sensitivity of pressure sensor can reach $15.8 \mathrm{~nm} / \mathrm{GPa}$. The results of simulation give an important step to achieve a monolithic integration of pressure sensor.

\section{References}

[1] J.D. Joannopoulos, S.G. Johnson, J.N. Winn, R.D. Meade, Photonic Crystals: Molding the Flow of Light, 2nd ed., Princeton University Press, Princeton (NJ) 2008.

[2] A. Hocini, A. Harhouz, J. Nanophoton. 10, 016007 (2016).

[3] A. Harhouz, A. Hocini, J. Electromagn. Wave Appl. 29, 659 (2015).

[4] T. Stomeo, M. Grande, A. Qualtieri, A. Passaseo, A. Salhi, M. De Vittorio, D. Biallo, A. D'orazio, M. de Sario, V. Marrocco, V. Petruzzelli, F. Prudenzano, Microelectron. Eng. 84, 1450 (2007).

[5] A. Kahlouche, A. Hocini, D. Khedrouche, J. Comp. Electron. 13, 490 (2014).

[6] A.R. Goni, K. Syassen, M. Cardona, Phys. Rev. B 41, 10104 (1990).

[7] H.K. Mao, Science 200, 1145 (1978).

[8] M. Kobayashi, T. Nagahama, Y. Nisida, in: Proc. 18th Int. Conf. on the Physics of Semiconductors, Stockholm 1986, Ed. O.Engstrom, World Scientific, Singapore 1986.

[9] Shangbin Tao, Deyuan Chen, Juebin Wang, Jing Qiao, Yali Duan, Photon. Sens. 6, 137 (2016). 